\title{
BMJ Open Regional South Australia Health (RESONATE) survey: study protocol
}

\author{
Matthew J Leach, ${ }^{1}$ Martin Jones, ${ }^{2}$ Marianne Gillam, ${ }^{1}$ Esther May ${ }^{3}$
}

To cite: Leach MJ, Jones M, Gillam M, et al. Regional South Australia Health (RESONATE) survey: study protocol. BMJ Open 2018;8:e019784. doi:10.1136/ bmjopen-2017-019784

- Prepublication history for this paper is available online. To view these files, please visit the journal online (http://dx.doi. org/10.1136/bmjopen-2017019784).

Received 25 September 2017 Revised 2 February 2018 Accepted 26 February 2018

\begin{abstract}
Introduction Access to quality healthcare services is considered a moral right. However, for people living in regional locations, timely access to the services that they need may not always be possible because of structural and attitudinal barriers. This suggests that people living in regional areas may have unmet healthcare needs. The aim of this research will be to examine the healthcare needs, expectations and experiences of regional South Australians.

Methods and analysis The Regional South Australia Health (RESONATE) survey is a cross-sectional study of adult health consumers living in any private or non-private dwelling, in any regional, rural, remote or very remote area of South Australia and with an understanding of written English. Data will be collected using a 45-item, multidimensional, self-administered instrument, designed to measure healthcare need, barriers to healthcare access and health service utilisation, attitudes, experiences and satisfaction. The instrument has demonstrated acceptable psychometric properties, including good content validity and internal reliability, good test-retest reliability and a high level of acceptability. The survey will be administered online and in hard-copy, with at least 1832 survey participants to be recruited over a 12-month period, using a comprehensive, multimodal recruitment campaign.

Ethics and dissemination The study has been reviewed and approved by the Human Research Ethics Committee of the University of South Australia. The results will be actively disseminated through peer-reviewed journals, conference presentations, social media, broadcast media, print media, the internet and various community/ stakeholder engagement activities.
\end{abstract}

\section{BACKGROUND}

Almost $46 \%$ of the world's population live in regional, rural, remote or very remote areas (hereafter referred to as regional); these regions are often characterised by much lower population densities (ie, global mean of 30.3 people per square kilometre) relative to urban areas (ie, global mean of 1109.6 people per square kilometre). ${ }^{1}$ In Australia, close to $33 \%$ of the nation's population live in regional areas, with an estimated population density as low as 10.33 people per square kilometre. ${ }^{23}$ The wide dispersion of the regional Australian population creates a number of challenges for healthcare delivery;
Strengths and limitations of this study

- This study will represent the largest survey ever conducted to examine the healthcare needs, expectations and experiences of regional South Australians.

- The use of non-probability sampling, while economically and logistically advantageous, will elevate the risk of self-selection bias.

- Multiple strategies will be put in place to mitigate the risk of sampling, undercoverage, recruitment and participation bias as well as measurement error.

for instance, timely access to the services that regional Australians need may not always be possible. The complexity of the Australian healthcare system (ie, the complex split of funding and responsibility across federal, state and territory governments, and across public and private sectors) amplifies the problem by creating additional challenges to the coordination, integration and continuity of healthcare services, especially for people living with chronic, comorbid conditions and in regional locations. ${ }^{4}$

In Australia, and internationally, there is a mounting body of evidence supporting the view that conventional healthcare services are struggling to meet the healthcare needs of consumers, particularly those with chronic health conditions and those living in regional areas. $^{5-11}$ The literature identifies some reasons why health consumer's needs are perhaps not being met. These barriers and enablers of healthcare utilisation can be broadly represented under two themes: structural factors (ie, accessibility, cost, time/availability, convenience) and attitude (ie, not needing medical support, stigma, improving symptoms, poor relationship with healthcare provider). ${ }^{1-15}$ For regional communities, these factors can be prominent obstacles to healthcare access. ${ }^{16-18}$

Andersen and Newman ${ }^{19}$ takealess simplistic view of health service use by viewing these determinants through a behavioural lens. Their construct, the Anderson Behavioural Model of health service use, identifies four 
key drivers of healthcare utilisation: predisposing factors (ie, prevailing conditions that predispose an individual to use a health service), enabling factors (ie, circumstances that either facilitate or hinder health service use), need factors (ie, actual or perceived need for health services) and personal health practices (ie, behaviours that influence health status). While many studies have used the Anderson Behavioural Model to investigate the use of health services, the range of variables reported to date has been limited and highly variable. ${ }^{20}$ There is also a need to better understand how these determinants of healthcare utilisation differ across populations (eg, between regional areas); the study described herein aims to address these knowledge gaps.

The impact of unmet healthcare need (ie, the difference between services required and services received ${ }^{10}$ at a systems level is not entirely clear. Several studies indicate that perceived unmet healthcare need is associated with higher rates of hospital admission, longer lengths of stay and more frequent visits to emergency departments ${ }^{21-23}$; however, the evidence is not consistent. ${ }^{24}$ Other studies suggest that those expressing an unmet health need access healthcare services less frequently ${ }^{6}$; this could have potential implications for consumer morbidity and mortality due to deficits in disease screening, monitoring, maintenance and risk reduction. ${ }^{25}$

At the individual level, the impact of unmet healthcare need can be substantial. Findings from several studies support an association between unmet health need and poorer quality of life, ${ }^{26-28}$ worse mental health ${ }^{28}$ and psychological distress. ${ }^{26}$ Although the direction of this association has yet to be determined, it does suggest that many healthcare systems have failed to some degree in meeting health consumer needs.

The significant implications of unmet consumer need signify the importance of furthering our understanding of the needs of health consumers; this is particularly evident in regional populations where there are considerable barriers to healthcare access, as well as a large health workforce maldistribution; as is the case in regional Australia. A more detailed exploration of the determinants of health service utilisation at a state/territory level may help to discern these needs. A population of particular importance is regional South Australia, which has one of the highest rates of chronic disease, comorbidity, psychological distress and fair/poor self-assessed health status of any state or territory of Australia. ${ }^{29}$ In addressing the abovementioned points, the proposed project will be the first known study to explore regional South Australian expectations and experiences in using diverse conventional and complementary healthcare services, with a view to better understand the healthcare needs of this population.

\section{METHODS}

\section{Study design}

The Regional South Australia Health (RESONATE) survey employs a cross-sectional study design. An overview of the study procedures, from questionnaire development through to the reporting of survey findings, is illustrated in figure 1.

\section{Aim and objectives}

RESONATE aims to examine the healthcare needs, expectations and experiences of regional South Australians. The objectives of the study will be to:

1. Determine the 12-month/lifetime prevalence of health conditions (ie, diagnosed or treated by a health professional), surgical procedures and multimorbidity (measured using the multiple chronic condition index ${ }^{30}$ ) among persons living in regional South Australia.

2. Examine the extent to which health services/treatments were used (ie, frequency of use in the previous 12 months) by persons living in regional South Australia.

3. Identify the information resources (eg, internet, friend, health provider) used in the previous 12 months to inform a person's decision to use a health service/treatment in regional South Australia.

4. Identify the extent (using a 4-point frequency scale) to which structural (eg, cost, distance) and attitudinal (eg, stigma) barriers prevent persons from accessing health services/treatments in regional South Australia.

5. Ascertain the experiences (ie, lifetime prevalence of adverse events, miscommunication, misunderstanding and disrespect, using a 4-point frequency scale) of persons living in regional South Australia with various health services/treatments.

6. Determine the attitudes of persons living in regional South Australia towards various health services/treatments (ie, perceptions of the roles, values, accessibility and quality of a heath care service, using a 5-point Likert scale).

7. Determine the degree (using a 5-point Likert scale) to which persons living in regional South Australia are satisfied with the quality of health services/treatments they have received.

8. Identify the determinants of health service utilisation, expectations and experiences among persons living in regional South Australia.
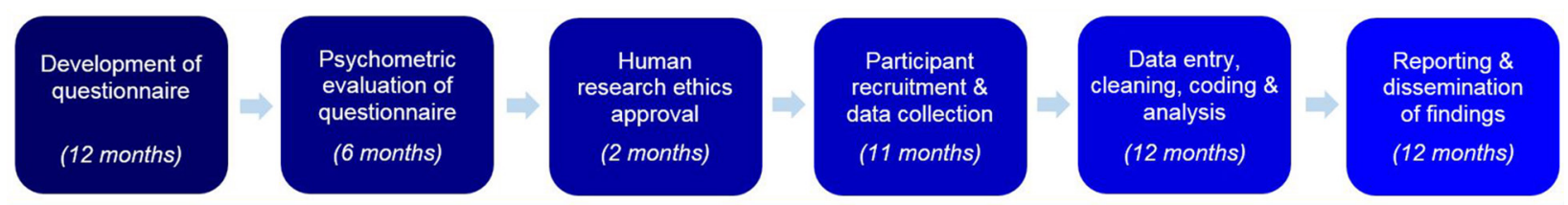

Figure 1 Flow chart of the Regional South Australia Health survey. 


\section{Sample and setting}

The study will use non-probability (self-selection) sampling for economic and logistical reasons. The sample will comprise adult health consumers (ie, a person over the age of 18 years who has used a healthcare service or received any health intervention within the last 12 months) living in any private or non-private dwelling, in any regional, rural, remote or very remote area of South Australia (a region that covers $99.7 \%$ of the land area of the state ${ }^{3}$ ). Participants also must be able to read and understand written English, comprehend the information provided and have either a fixed address (for delivery of the hard-copy version of the survey) or access to the internet (to access the online version of the survey). Excluded will be people with severe cognitive impairment, severe vision impairment and those not able to provide consent. Based on a target population of 290290 adults, the study will need to survey at least 1832 persons; this is based on a $\pm 3 \%$ margin of error at the $99 \%$ confidence level.

\section{Questionnaire}

The consumer utilisation, expectations and experiences of healthcare instrument (CONVERSATIONS) is a multidimensional, self-administered questionnaire designed to measure health service utilisation, needs, expectations and experiences. The development, validation and description of the instrument are detailed below.

\section{Development}

Development of the questionnaire was an iterative process that began with an extensive search of the health motivation literature, the interrogation of pertinent surveys $^{30-35}$ and informal consultation with clinicians, researchers and consumers. This generated a large pool of potential survey items. Using the Andersen behavioural model of health service use ${ }^{19}$ as the conceptual framework for the survey, potential questions were placed into one of four categories: predisposing factors, enabling factors, need factors and personal health practices (figure 2 ). The research team reviewed the items under each category to ensure questions adequately captured the construct of the framework (ie, to confirm face validity), questions were clear in their meaning, response items were comprehensive and any duplicate/overlapping items were removed. The list of items, comprising a combination of open questions (ie, free text boxes) and closed questions (ie, Likert scales, dichotomous items and nominal items), were then reordered to improve the flow of the survey, to simplify data analysis and to be more meaningful to respondents.

\section{Validation}

The preliminary 51-item CONVERSATIONS underwent a two-stage psychometric evaluation. The first stage recruited a purposive sample of nine international academics with expertise in survey design and/or health service utilisation, as well as a track record in regional health research, to assess the content validity of the survey. Academics were identified through online staff directories of major Australian and international Universities. The sample comprised 3 academics from Australia, 2 from the USA and 1 each from Spain, New Zealand, the UK and Israel, of whom 5/9 were female. Using the

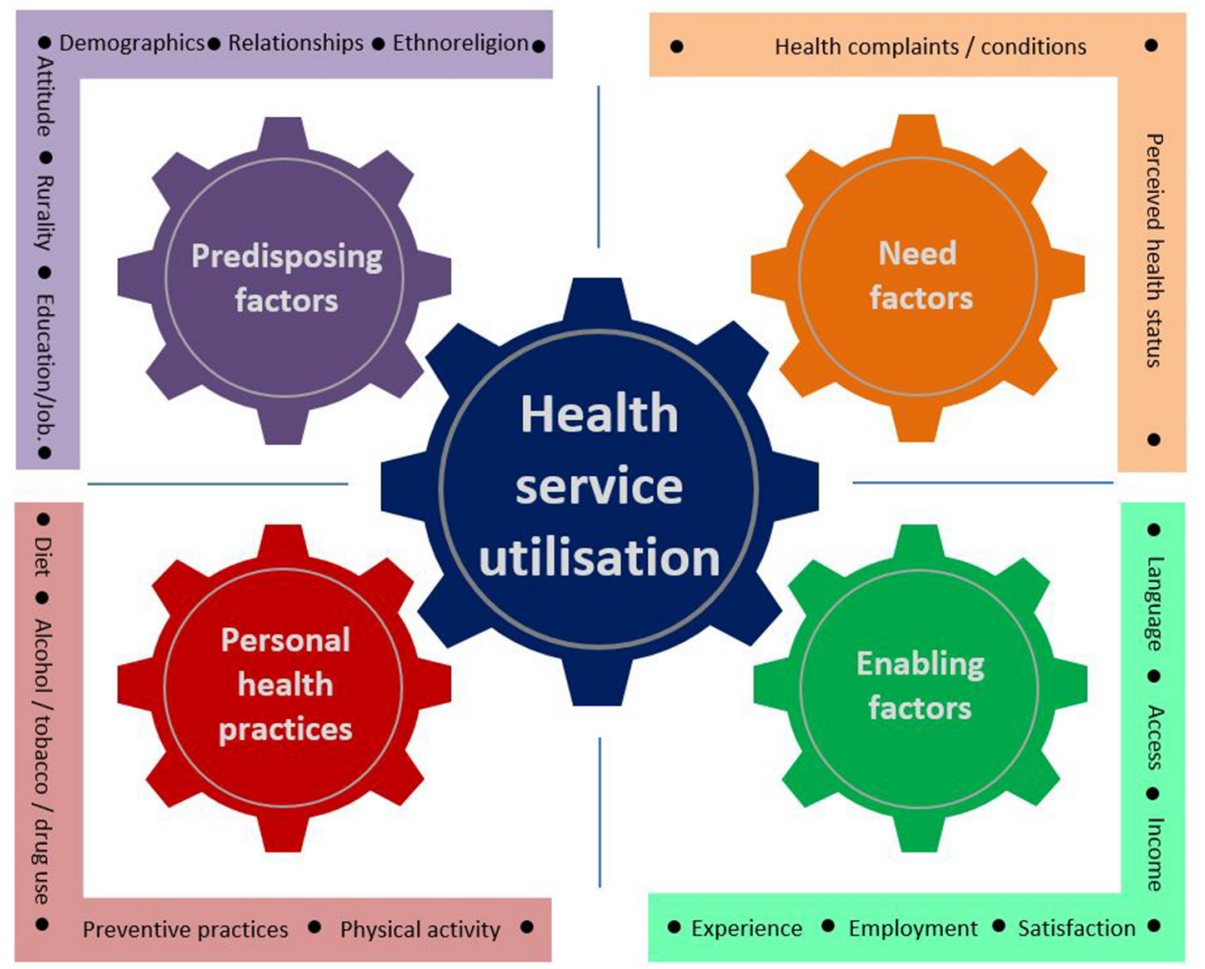

Figure 2 Conceptual framework of the CONVERSATIONS. 
method described by Polit and Hungler, ${ }^{36}$ respondents indicated the relevance of each survey item by assigning one of four responses to each item: (1) question is relevant, (2) question is relevant but needs minor alteration, (3) question is relevant but needs major alteration and (4) question is not relevant to the survey construct (ie, health service need). The mean percentage of items with a score of 3 or 4 was calculated (ie, agreement of relevancy) for each subsection of the survey, and for the survey overall, with good content validity defined as a level of agreement of $80 \%$ or above ${ }^{3637}$ After the removal of seven irrelevant questions, the CONVERSATIONS survey was shown to have good scale-level content validity (mean $85.3 \% \pm \mathrm{SD}$ $13.1 \%$ ), and good subscale-level content validity (part A $84.1 \% \pm 17.9 \%$; part B $86.7 \% \pm 8.6 \%$; part C $81.8 \% \pm 12.1 \%$; part D $88.8 \% \pm 9.6 \%$; part E $88.5 \% \pm 0.9 \%)$. Of the retained questions, 16 items underwent minor editorial changes based on expert feedback.

In the second stage of evaluation, a purposive sample of 16 health consumers, of various age groups, and diverse cultural, educational and socioeconomic backgrounds (including one-third with lived experience in regional Australia), were invited to complete the CONVERSATIONS, on two separate occasions, 2 weeks apart. The purposive sample was identified through the research team's social network, and comprised 11 females and 5 males, aged between 21 and 66 years (mean age $42.9 \pm 10.2$ years), of whom 12 resided in Australia, 3 in the UK and 1 in Singapore. Data from the baseline survey were used to assess the acceptability (ie, frequency of missing data, completion time) and internal consistency (ie, Cronbach's $\alpha$ ) of the instrument. The baseline and week 2 data were used to measure test-retest reliability (using the intraclass correlation coefficient (absolute agreement, two-way mixed-effects model) for scale and ordinal data, and Cohen's Kappa for nominal data).

The analysis revealed a median completion time of 20 (IQR 15, 30) minutes, and a high level of acceptability, with 15/16 (93.8\%) participants submitting a fully completed survey at baseline. The 6 -item experience subscale and 16-item attitude subscale of the instrument demonstrated good to excellent internal reliability, ${ }^{36}$ with values reported as follows: experience of conventional treatments (a component of part $\mathrm{C}$ : $\alpha=0.92$ ); experience of complementary and alternative medicine (CAM) treatments (a component of part D: $\alpha=0.88$ ); attitude towards conventional treatments (a component of part $\mathrm{C}: \alpha=0.90$ ) and attitude towards CAM treatments (a component of part D: $\alpha=0.88$ ). There was also good to excellent agreement between baseline and week 2 scores for three out of five parts of the instrument (mean reliability coefficients: part A $0.962,95 \%$ CI 0.950 to 0.973 ; part B 0.827 , $95 \%$ CI 0.738 to 0.917 ; part C $0.768,95 \%$ CI 0.701 to 0.834 ), and moderate agreement between scores for two parts (mean reliability coefficients: part D $0.699,95 \%$ CI 0.603 to 0.795 ; part E $0.741,95 \%$ CI 0.408 to 1.000 ). Overall, the CONVERSATIONS demonstrated good test-retest reliability (overall mean reliability coefficient: 0.799 , $95 \%$ CI 0.749 to 0.849$).{ }^{38}$

Given the multidimensionality and multidisciplinary nature of the survey, as well as the self-administered design, there was no similar instrument for which the CONVERSATIONS could be compared against; as such, it was not possible to measure convergent validity.

\section{Description}

The final instrument was a 44-item questionnaire divided into five sections: (i) demographic characteristics (part A; 16 items, including age, sex, level of education, marital status, caregiver status, religion, English language proficiency, health literacy, country of birth, number of dependent children, regional classification, employment status, occupation, annual household income, current postcode, years lived in postcode), (ii) health status and lifestyle (part $\mathrm{B} ; 10$ items, including overall health rating, diagnosed health conditions, surgical history, sedentary duration, dietary intake, level of physical activity, alcohol consumption, smoking status, illicit drug use, health screening activity), (iii) use of conventional/mainstream health services (part C; 8 items, including 12-month/lifetime use of conventional health services, frequency of visits to conventional health providers, satisfaction with the quality of care received by conventional health providers, utilisation of conventional health treatments/services, information resources impacting the decision to use conventional healthcare services, experience with using conventional health services, barriers to accessing conventional health services, attitude towards conventional health services), (iv) use of complementary/alternative/natural health and self-prescribed services (part D; 9 items, including 12-month/lifetime use of complementary health services, frequency of visits to complementary health providers, satisfaction with the quality of care received by complementary health providers, utilisation of complementary and self-prescribed health treatments/services, information resources impacting the decision to use complementary healthcare services, experience with using complementary health services, barriers to accessing complementary health services, attitude towards complementary health services) and (v) other (part E; 1 item, measuring preferred mix of health services). A hard-copy version and online version (using the SurveyMonkey platform) of the CONVERSATIONS have been generated for this study.

\section{Recruitment/procedures}

The project will implement a comprehensive, multimodal recruitment campaign, and in accordance with a community-based participatory approach, will involve extensive community engagement. The strategies that will be employed are outlined in table 1 . The majority of these strategies will direct participants to the project website, which will contain further information about the study, the participant information sheet, a web enquiry form and a link to the online survey. Participants who 
Table 1 Recruitment strategies for the RESONATE survey

\begin{tabular}{|c|c|c|}
\hline Category & Strategy & Platform/agency/medium \\
\hline \multirow[t]{2}{*}{ Social media } & Social media advertising & Facebook ads (targeting a regional SA audience) \\
\hline & $\begin{array}{l}\text { Social media posts (ie, study } \\
\text { information/invitations) }\end{array}$ & Facebook pages, Linkedln, Twitter \\
\hline \multirow[t]{2}{*}{ Broadcast media } & Region-specific media releases & All television and radio stations in regional SA \\
\hline & Television classified advertising & All television stations based in regional SA \\
\hline \multirow{2}{*}{ Print media } & Study flyers & $\begin{array}{l}\text { All local councils, public libraries and community agencies in } \\
\text { regional SA }\end{array}$ \\
\hline & Newsletter articles & $\begin{array}{l}\text { All local councils, community groups, sporting groups, primary } \\
\text { health networks and industry groups in regional SA }\end{array}$ \\
\hline \multirow[t]{3}{*}{$\begin{array}{l}\text { Community } \\
\text { engagement }\end{array}$} & Public lectures & $\begin{array}{l}\text { All University of South Australia Department of Rural Health major } \\
\text { training sites; community groups }\end{array}$ \\
\hline & Stakeholder group meetings & $\begin{array}{l}\text { Country SA primary health network regional committees; local } \\
\text { councils }\end{array}$ \\
\hline & Community/public events & Community fairs; conferences; exhibitions; shopping centre displays \\
\hline
\end{tabular}

RESONATE, Regional South Australia Health; SA, South Australia.

cannot (or prefer not to) complete the survey online will be advised to contact the research department, using the toll-free telephone number provided, to have the participant information sheet, survey and reply-paid envelope posted out to them. To facilitate recruitment, all participants who opt in will be entered into a draw to win one of 20×AU \$50 gift cards. Recruitment for the survey will take place between April 2017 and March 2018.

\section{Planned analysis}

Data from hard-copy surveys will be directly entered into the online survey by the research team. On completion of the project, data will be exported from the SurveyMonkey platform into SPSS (V.24) for data cleaning and statistical analysis. Missing data will be prevented by enacting forced survey responses. Multiple responses from single participants will be managed using the deduplication procedure for online surveys described by Konstan et al. ${ }^{39}$ In brief, all responses will be screened for duplicate internet service provider (ISP) entries (including the first three quadrants of an ISP address). Any duplicate ISP entries that report matching demographic data (ie, age, sex, highest education and marital status) will be considered a duplicate response and subsequently excluded from the analysis (with only the first dated entry retained). Categorical data will be descriptively analysed using frequency distributions and percentages. Measures of central tendency and variability will be used for continuous data where values are normally distributed, whereas medians and the IQR will be used to describe data that is not normally distributed. Differences between groups will be assessed using independent samples t-tests or Mann-Whitney U test (for continuous variables), $\mathrm{X}^{2}$ test (for categorical variables) and analysis of variance or Kruskal-Wallis (where there are more than two groups). Independent predictors of health service utilisation (ie, frequency of visits to health providers), expectations (ie, healthcare attitude score) and experiences (ie, healthcare experience score) will be identified using regression analysis. The representativeness of the sample to the base population will be cross-checked against regional South Australia demographic data derived from the 2016 Australian population census. To mitigate the self-selection bias, the survey sample distribution will be adjusted by applying weights to the age, sex and location distribution of the regional South Australian population; these weights will be based on 2016 Australian population census data.

\section{DISCUSSION}

Health inequalities and inadequate health service provision are major concerns facing regional Australia. In fact, living in regional locations of Australia is associated with poorer health outcomes, increased chronic disease mortality and lower life expectancy when compared with living in metropolitan locations. ${ }^{40-43}$ An important first step in addressing these health status disparities is understanding the healthcare needs of the regional population. The RESONATE survey will explore these needs in detail, 
as well as gain new insights into the healthcare expectations and experiences of people living in regional South Australia.

In addition to shedding new light on the healthcare needs of regional South Australians, the findings of this research will make an important contribution to future health services planning. Using the needs-based health workforce planning framework, the healthcare needs of this population can be mapped against best practice care to estimate total health workforce requirements. ${ }^{44}$ This model has already been applied to regional populations with diabetes ${ }^{45}$ and mental illness, ${ }^{46}$ and takes a far more 'richer perspective on population needs' than other workforce planning approaches. ${ }^{44}$ Given the considerable health workforce maldistribution in regional South Australia, ${ }^{47-49}$ this is an important next step for this research.

If the RESONATE study can reach, or even exceed its target of 1832 participants, it will represent the largest health survey conducted in regional South Australia. Importantly, the study will complement the results of other large studies of regional South Australians, including the Australian Bureau of Statistics National Health Survey (estimated $\mathrm{n}=818)^{30}$ and the National Regional Well-being Survey $(\mathrm{n}=1126),{ }^{50}$ by reporting unique insights into the regional South Australian population not yet available. Furthermore, the large sample will enable meaningful subgroup analyses to be performed to better inform local policy and strategy (eg, comparing needs between regions and statistical areas). ${ }^{51}$ The latter is a particularly important point, as the project is underpinned by a community-based participatory approach, whereby local communities will be actively involved in project promotion and implementation, as well as the dissemination and translation of research findings; this will ensure the research informs and facilitates meaningful change at the local level. ${ }^{52}$

Despite its strengths, the RESONATE study does have a limitation-its susceptibility to self-selection bias. While it is not possible to eliminate this bias entirely due to the use of non-probability (self-selection) sampling, the study has put in place multiple measures to help mitigate this risk and the risk of other biases. These strategies include the implementation of a comprehensive multimodal recruitment campaign with extensive reach to the regional SA population (to minimise undercoverage bias); intensive community engagement (to maximise widespread community participation and reduce sampling bias); the provision of alternative survey administration methods (to mitigate recruitment bias); the use of an instrument with acceptable psychometric properties (to reduce measurement error) and the weighting of sample data (to adjust for an unrepresentative sample) ${ }^{53}$

In summary, RESONATE will represent the largest health survey ever conducted in regional South Australia. The study will further our understanding of the state of health of regional South Australia, and will impart new insights into the health service experiences, utilisation and expectations of this population. Accordingly, the findings of this research will help us to better understand the healthcare needs of regional South Australians. An important next step of this research will be to map these needs against existing health workforce supply to enable policy makers, healthcare providers, researchers and educationalists to identify the health workforce required to better support the health of regional South Australians.

\section{ETHICS AND DISSEMINATION}

The study will be conducted in accordance with the National Health and Medical Research Council national statement on ethical conduct in research, as well as the approved study protocol. A detailed participant information sheet will preface each survey, with voluntary completion of the survey implying informed consent to participate. No personally identifiable information will be collected in order to maintain the anonymity of the survey.

The findings of the survey will be communicated using a comprehensive dissemination strategy. The strategy will use various forms of media to reach out to a diverse range of stakeholder groups and individuals, at the local, national and international level; this will include the use of academic media (ie, peer-reviewed journal articles, national and international conference presentations), social media (ie, Facebook, Twitter), print media (ie, newspaper), broadcast media (ie, radio, television), the internet (ie, links to study reports on the Department of Rural Health website), electronic and postal mail (ie, posting of study findings to participants and stakeholders) and community/stakeholder engagement activities (ie, community forums, stakeholder meetings).

Contributors MJL conceptualised the project and drafted the manuscript. MJ, MG and EM reviewed and edited the manuscript. All authors read and approved the final manuscript.

Funding This research will be supported by infrastructure provided by the Department of Rural Health, University of South Australia.

Competing interests None declared.

Patient consent Not required.

Ethics approval The study was approved by the Human Research Ethics Committee of the University of South Australia (Protocol ID: 0000034611 ). Participants will be informed about the study via the survey cover sheet and project website. Informed consent will be implied through completion of the survey.

Provenance and peer review Not commissioned; externally peer reviewed.

Open Access This is an Open Access article distributed in accordance with the Creative Commons Attribution Non Commercial (CC BY-NC 4.0) license, which permits others to distribute, remix, adapt, build upon this work non-commercially, and license their derivative works on different terms, provided the original work is properly cited and the use is non-commercial. See: http://creativecommons.org/ licenses/by-nc/4.0/

(c) Article author(s) (or their employer(s) unless otherwise stated in the text of the article) 2018. All rights reserved. No commercial use is permitted unless otherwise expressly granted. 


\section{REFERENCES}

1. The World Bank. World bank open data. 2017 http://data.worldbank. org/ (accessed 10 Aug 2017).

2. Australian Bureau of Statistics. Regional Population Growth, Australia, 2016. Cat. No. 3218.0. Canberra, Australia: Australian Bureau of Statistics, 2017.

3. Australian Bureau of Statistics. ABS.Stat. Canberra, Australia: Australian Bureau of Statistics, 2017.

4. Organisation for Economic Co-operation \& Development (OECD). OECD health policy overview: health policy in Australia. Paris, France: OECD, 2015.

5. Ahern T, Gardner A, Courtney M. Exploring patient support by breast care nurses and geographical residence as moderators of the unmet needs and self-efficacy of Australian women with breast cancer: Results from a cross-sectional, nationwide survey. Eur J Oncol Nurs 2016;23:72-80.

6. Colman E, Missinne S, Bracke P. The role of perceived helpfulness in predicting subjective unmet need and the frequency of health care use. Arch Psychiatr Nurs 2014;28:43-9.

7. Dezetter A, Duhoux A, Menear M, et al. Reasons and determinants for perceiving unmet needs for mental health in primary care in Quebec. Can J Psychiatry 2015;60:284-93.

8. Ghuman SJ, Brackbill RM, Stellman SD, et al. Unmet mental health care need 10-11 years after the 9/11 terrorist attacks: 2011-2012 results from the World Trade Center Health Registry. BMC Public Health 2014;14:491-9.

9. Ou L, Chen J, Hillman K. Socio-demographic disparities in the utilisation of general practice services for Australian children Results from a nationally representative longitudinal study. PLoS One 2017;12:e0176563.

10. Pappa E, Kontodimopoulos N, Papadopoulos A, et al. Investigating unmet health needs in primary health care services in a representative sample of the Greek population. Int J Environ Res Public Health 2013;10:2017-27.

11. Ronksley PE, Sanmartin C, Campbell DJ, et al. Perceived barriers to primary care among western Canadians with chronic conditions. Health Rep 2014;25:3-10.

12. Andrade LH, Alonso J, Mneimneh Z, et al. Barriers to mental health treatment: results from the WHO World Mental Health surveys. Psychol Med 2014;44:1303-17.

13. Allen $\mathrm{H}$, Wright $\mathrm{BJ}$, Harding $\mathrm{K}$, et al. The role of stigma in access to health care for the poor. Milbank Q 2014;92:289-318.

14. Monger M. Stigma: barrier to quality of life and health care. HIV Clin 2011;23:1,4-5.

15. Sheikh-Mohammed M, Macintyre CR, Wood NJ, et al. Barriers to access to health care for newly resettled sub-Saharan refugees in Australia. Med J Aust 2006;185:594-7.

16. Hull MJ, Fennell KM, Vallury $\mathrm{K}$, et al. A comparison of barriers to mental health support-seeking among farming and non-farming adults in rural South Australia. Aust J Rural Health 2017;25:347-53.

17. Stewart $H$, Jameson JP, Curtin L. The relationship between stigma and self-reported willingness to use mental health services among rural and urban older adults. Psychol Serv 2015;12:141-8.

18. Syed ST, Gerber BS, Sharp LK. Traveling towards disease: transportation barriers to health care access. J Community Health 2013;38:976-93.

19. Andersen R, Newman JF. Societal and Individual Determinants of Medical Care Utilization in the United States. Milbank Q 2005;83:Online-only.

20. Babitsch B, Gohl D, von Lengerke T. Re-revisiting Andersen's Behavioral Model of Health Services Use: a systematic review of studies from 1998-2011. Psychosoc Med 2012;9:Doc11.

21. Bindman AB, Grumbach K, Osmond D, et al. Preventable hospitalizations and access to health care. JAMA 1995;274:305-11.

22. McCusker J, Roberge D, Lévesque JF, et al. Emergency department visits and primary care among adults with chronic conditions. Med Care 2010;48:972-80.

23. Zuckerman S, Shen YC. Characteristics of occasional and frequent emergency department users: do insurance coverage and access to care matter? Med Care 2004;42:176-82.

24. Ronksley PE, Sanmartin C, Quan H, et al. Association between perceived unmet health care needs and risk of adverse health outcomes among patients with chronic medical conditions. Open Med 2013;7:e21-30.

25. Ayanian JZ, Weissman JS, Schneider EC, et al. Unmet health needs of uninsured adults in the United States. JAMA 2000;284:2061-9.

26. Hansen DG, Larsen PV, Holm LV, et al. Association between unmet needs and quality of life of cancer patients: a population-based study. Acta Oncol 2013;52:391-9.
27. Slade M, Leese M, Cahill S, et al. Patient-rated mental health needs and quality of life improvement. Br J Psychiatry 2005;187:256-61.

28. Smith AW, Parsons HM, Kent EE, et al. Unmet Support Service Needs and Health-Related Quality of Life among Adolescents and Young Adults with Cancer: The AYA HOPE Study. Front Oncol 2013;3:75.

29. Australian Bureau of Statistics. National Health Survey: First Results, 2014-15 Cat. No. 4364.0.55.001. Canberra: Australian Bureau of Statistics, 2015.

30. Falci L, Shi Z, Greenlee H. Multiple Chronic Conditions and Use of Complementary and Alternative Medicine Among US Adults: Results From the 2012 National Health Interview Survey. Prev Chronic Dis 2016;13:150501.

31. Busato A, Dönges A, Herren S, et al. Health status and health care utilisation of patients in complementary and conventional primary care in Switzerland--an observational study. Fam Pract 2006;23:116-24.

32. Chao MT, Wade C, Kronenberg F, et al. Women's reasons for complementary and alternative medicine use: racial/ethnic differences. J Altern Complement Med 2006;12:719-20.

33. Simões-Wüst AP, Rist L, Dettling M. Self-reported health characteristics and medication consumption by CAM users and nonusers: a Swiss cross-sectional survey. J Altern Complement Med 2014;20:40-7.

34. Sirois FM. Motivations for consulting complementary and alternative medicine practitioners: a comparison of consumers from 1997-8 and 2005. BMC Complement Altern Med 2008;8:16.

35. Women's Health Australia. Australian Longitudinal Study on Women's Health: Surveys. 2015 http://www.alswh.org.au/for-researchers/ surveys\# (accessed 23 Jul 2015).

36. Polit D, Hungler B. Nursing Research: Principles and Methods. seventh edn. Philadelphia, USA: Lippincott, 2004.

37. Davis LL. Instrument review: Getting the most from a panel of experts. Applied Nursing Research 1992;5:194-7.

38. Koo TK, Li MY. A Guideline of Selecting and Reporting Intraclass Correlation Coefficients for Reliability Research. J Chiropr Med 2016;15:155-63.

39. Konstan JA, Simon Rosser BR, Ross MW, et al. The Story of Subject Naught: A Cautionary but Optimistic Tale of Internet Survey Research. Journal of Computer-Mediated Communication 2005;10:00.

40. Australian Institute of Health and Welfare (AIHW). Rural, regional and remote health - indicators of health system performance. Rural Health Series no. 10, cat. No. PHE103. Canberra, Australia: AlHW, 2008.

41. Beckmann KR, Bennett A, Young GP, et al. Sociodemographic disparities in survival from colorectal cancer in South Australia: a population-wide data linkage study. BMC Health Serv Res 2016;16:24.

42. Chondur R, Li SQ, Guthridge S, et al. Does relative remoteness affect chronic disease outcomes? Geographic variation in chronic disease mortality in Australia, 2002-2006. Aust N Z J Public Health 2014;38:117-21.

43. Fox $P$, Boyce $A$. Cancer health inequality persists in regional and remote Australia. Med J Aust 2014;201:445-6.

44. Segal L, Leach MJ. An evidence-based health workforce model for primary and community care. Implement Sci 2011;6:93.

45. Segal L, Leach MJ, May E, et al. Regional primary care team to deliver best-practice diabetes care: a needs-driven health workforce model reflecting a biopsychosocial construct of health. Diabetes Care 2013;36:1898-907.

46. Furber G, Segal L, Leach M, et al. Preventing mental illness: closing the evidence-practice gap through workforce and services planning. BMC Health Serv Res 2015;15:283.

47. Department of Health. The national health workforce dataset (NHWDS): Physiotherapy 2015. Canberra, Australia: Department of Health, 2015.

48. Department of Health. Nurses and midwives 2016 fact sheet. Canberra, Australia: Department of Health, 2017.

49. Tennant M, Kruger E, Shiyha J. Dentist-to-population and practiceto-population ratios: in a shortage environment with gross maldistribution what should rural and remote communities focus their attention on? Rural Remote Health 2013;13:2518.

50. Centre for Research and Action in Public Health. 2015 regional wellbeing survey data tables: South Australia - natural resource management regions. Canberra, Australia: University of Canberra, 2016.

51. De Vaus D. Surveys in social research. sixth edn. Oxon, UK: Routledge, 2014.

52. Hacker K. Community-based participatory research. Thousand Oaks USA: Sage, 2013.

53. Keeble C, Law GR, Barber S, et al. Choosing a method to reduce selection bias: a tool for researchers. Open J Epidemiol 2015;05:155-62. 